\title{
Aspectos da biologia de Digitaria insularis resistente ao glyphosate e implicações para o seu controle ${ }^{1}$
}

\author{
Biology aspects of Digitaria insularis resistant to glyphosate and implications for \\ its control
}

\author{
Alexandre Gemelli ${ }^{2}$; Rubem Silvério de Oliveira Jr. ${ }^{3}$; Jamil Constantin ${ }^{3}$; Guilherme Braga \\ Pereira Braz ${ }^{4}$; Talita Mayara de Campos Jumes ${ }^{5}$; Antônio Mendes de Oliveira Neto ${ }^{4}$; Hugo de \\ Almeida Dan ${ }^{4}$; Denis Fernando Biffe ${ }^{3}$
}

Resumo - O capim-amargoso (Digitaria insularis) é uma planta perene que tem a capacidade de emergir e se desenvolver praticamente o ano inteiro nas condições climáticas brasileiras. Uma vez estabelecida com a formação de rizomas, a dificuldade de controle dessa espécie aumenta muito. Com a recente confirmação da existência de biótipos resistentes à glyphosate os problemas se agravaram e o conhecimento da biologia dessa espécie é fundamental na elaboração de estratégias para o manejo químico do capim-amargoso. A presença dessa espécie deve ser monitorada nas lavouras durante a safra, safrinha e entressafra, pois o descaso com a ocorrência dessa planta na lavoura pode levar a um forte aumento na população de $D$. insularis e consequentemente a sérios prejuízos na produtividade e aumento nos custos de produção.

Palavras-chaves: capim-amargoso, resistência, controle químico

\begin{abstract}
Sourgrass (Digitaria insularis) is a perennial weed which has the ability to germinate, grow and develop during the entire year under Brazilian weather conditions. Once established with the formation of rhizomes, the difficulty of control greatly increases. With the recent confirmation of the existence of resistant biotypes to glyphosate, the problems became worse and knowledge on the biology of this species is critical to develop strategies for chemical management of sourgrass. The presence of this species should be monitored during the crop growing seasons (winter and summer) and also during off-season period, once neglecting the occurrence of this plant in the area can lead to a sharp increase in the population of Digitaria insularis and consequently to serious crop yield losses and increased production costs.
\end{abstract}

Keywords: sourgrass, resistance, chemical control

\footnotetext{
${ }^{1}$ Recebido para publicação em 20/04/2012 e aceito em 30/07/2012.

${ }^{2}$ Eng. Agr., Mestrando em Agronomia pela Universidade Estadual de Maringá (UEM). Av. Colombo 5790 - 87020 900 Maringá, PR - alexandregemelli@gmail.com;

${ }^{3}$ Professor Doutor do Departamento de Agronomia da Universidade Estadual de Maringá - Núcleo de Estudos avançados em Ciência das Plantas daninhas (NAPD/UEM);

${ }^{4}$ Eng. Agr., Doutorando em Agronomia pela Universidade Estadual de Maringá (UEM);

${ }^{5}$ Graduando em Agronomia da Universidade Estadual de Maringá (UEM).
} 


\section{Introdução}

O gênero Digitaria sp. compreende cerca de 300 espécies de plantas distribuídas em diferentes regiões do mundo, tanto de clima tropical quanto subtropical (Canto-Dorow, 2001). O capim-amargoso (Digitaria insularis (L.) Fedde) é uma espécie nativa de regiões tropicais e subtropicais da América, onde é frequentemente encontrado em pastagens, cafezais, pomares e em áreas ruderais como beira de estradas e terrenos baldios (Machado et al., 2008).

O Brasil é o país com maior diversidade de espécies do gênero Digitaria sp., sendo constatada a presença de 26 espécies nativas e de 12 exóticas. Entre estas espécies, atualmente, uma das que apresenta ampla distribuição geográfica é o capim-amargoso, ocorrendo na maioria dos ambientes favoráveis à agricultura, desde o continente asiático ao americano (Mondo et al., 2010). Digitaria insularis possui vários nomes comuns, destacando-se os seguintes no Brasil: capimamargoso, capim-flecha, capim-açú, capimpororó e milheto-gigante. $\mathrm{Na}$ língua inglesa o nome comum é sourgrass, que pode ser traduzido como capim-ácido, devido ao paladar ácido que esta planta apresenta quando ingerida.

Existem muitas sinonímias para Digitaria insularis, entre elas Andropogon insularis L.; Trichachne insularis (L.) Nees; Tricholaena insularis (L.) Griseb.; Valota insularis (L.) Chase; Digitaria insularis(L.) Mezex Ekman; Panicum leucophaeum Kunth, in Humb. \& Bonpl.; Acicarpa sacchariflora Raddi; Trichachne sacchariflora Nees; Digitaria sacchariflora (Nees) Henrard; Leptocoryphium penicilligerum Speg. E Panicum insulare (L.) G. Mey. var. sacchariflorum (Nees) Hack. Ex Stuckert. Contudo, o nome científico mais aceito é Digitaria insularis por ser o primeiro nome dado a esta espécie, sendo, portanto, o mais antigo (Canto-Dorow, 2001).
Nas últimas décadas, principalmente após o advento do sistema de plantio direto, esta espécie vem apresentando maior relevância dentro da agricultura brasileira, sendo este aumento de ocorrência relacionado às suas características de agressividade. Entre elas destaca-se a capacidade de formação de rizomas, que apesar de curtos são bem evidentes, formando notáveis touceiras (Clayton et al., 2006) e a capacidade de disseminação de propágulos (sementes) praticamente durante todo o verão (Lorenzi, 2000; Kissmann \& Groth, 1997). As sementes desta espécie são revestidas por muitos pêlos, os quais auxiliam sua dispersão a longas distâncias, o que, aliado ao grande percentual germinativo, permite que essa planta se dissemine com grande facilidade (Kissmann \& Groth, 1997).

Digitaria insularis é uma espécie de gramínea de ciclo perene com metabolismo fotossintético do tipo C4 (Kissmann \& Groth, 1997), porém apresenta crescimento inicial lento até 45 dias após a emergência (DAE). Dos 45 aos 105 DAE o seu crescimento é acelerado, apresentando aumento exponencial de matéria seca. Esse comportamento foi observado para raiz+rizoma, colmo e folha (folha+inflorescência). Na fase de crescimento exponencial, parte do incremento de massa seca das raízes a partir dos 45 DAE se deve à formação dos rizomas. Além disso, a emissão de inflorescências em $D$. insularis ocorre entre os 63 e 70 DAE (Machado et al., 2006).

Apesar de o capim-amargoso apresentar insensibilidade ao fotoperíodo para o estímulo ao florescimento, quanto maior é o fotoperíodo, mais rápida é a emissão da panícula e maior é o acúmulo de matéria seca por planta (Pyon et al., 1977). O acúmulo de massa seca de D. insularis descrito por Machado et al. (2006) (30,66 g por planta aos 98 DAE) é superior quando comparado com outra gramínea como B. plantaginea, a qual apresentou 23,87 g por planta aos 143 DAE (Carvalho et al., 2007). Porém esse acúmulo 
não é tão expressivo quanto o de uma espécie como Brachiaria brizantha cv. MG5 (53,6 g por planta aos 70 DAE), quando destinada à utilização como forrageira (Silva et al., 2005).

Em áreas onde há uso contínuo de glyphosate, constata-se que plantas originárias de sementes, quando jovens, são controladas facilmente por esse herbicida; contudo, quando elas se desenvolvem e formam rizomas, seu controle é dificultado. Dessa forma, infere-se que o melhor período para controle de $D$. insularis é até os $45 \mathrm{DAE}$, quando os rizomas ainda não foram formados (Machado et al., 2006).

As plantas de $D$. insularis provenientes de rizomas apresentam folhas com maior índice estomático, quando comparadas com plantas provenientes de sementes, para as duas faces da epiderme. Além disso, plantas provenientes de rizomas mostram lâmina foliar mais espessa, com epiderme das faces adaxial e abaxial apresentando o mesmo comportamento (Machado et al., 2008).

A maior espessura da folha é uma característica de plantas adaptadas à sombra (Castro, et al., 1987; Taiz \& Zeiger, 2004). A partir dessa constatação pode se esperar que as plantas de capim-amargoso oriundas da rebrota do rizoma sejam mais tolerantes ao sombreamento, e, portanto, à supressão de luz realizada pela cultura (controle cultural).

Enquanto isso, quando se trata de plantas oriundas de sementes no início de seu desenvolvimento, Pyon (1975) verificou a baixa capacidade competitiva de capimamargoso em relação à Panicum maximum e Cenchrus ciliaris em condições normais de luminosidade. E ainda, quando estas espécies se desenvolveram sob condições de até $60 \%$ de sombreamento, Digitaria insularis teve sua altura, número de perfilhos e matéria seca reduzida drasticamente em relação às demais espécies avaliadas. Porém, mesmo com a aplicação equivalente a $60 \mathrm{Kg}$ de nitrogênio por hectare, essa espécie não conseguiu competir com as demais.
Essas informações estão em concordância com o período de crescimento inicial lento descrito por Machado et al. (2006) e suportam a afirmação de que "o início do desenvolvimento de Digitaria insularis é o período de maior sensibilidade dessa planta daninha”, e, portanto, quando há a maior facilidade para a realização de seu controle.

Em condições de temperaturas constantes entre 20 e $30^{\circ} \mathrm{C}$ a germinação das sementes de capim-amargoso (Digitaria insularis) depende da presença de luz. Já em condições de temperaturas alternadas, entre 15 e $35^{\circ} \mathrm{C}$, a germinação é semelhante tanto na presença quanto ausência de luz, superando facilmente $90 \%$ de germinação após 10 dias (Pyon, 1975; Mondo et al., 2010). Além disso, quando submetidas ao fotoperíodo de 8 a 12 horas de luz, a germinação é mais rápida, mostrando índices de germinação próximos à $70 \%$ em 5 dias (Pyon, 1975).

A necessidade dessa variação de temperatura durante a germinação geralmente está associada com modificações na permeabilidade do tegumento das sementes à água e a trocas gasosas (Braccini, 2011). É possível ainda que a alternância de temperatura interfira no equilíbrio entre substâncias promotoras e inibidores de dormência em sementes, como a relação entre as formas do fitocromo, vermelho e vermelho-distante (Mondo et al., 2010).

A emergência das plântulas de Digitaria insularis é afetada pela profundidade do solo na qual as sementes são posicionadas. Maior porcentagem e maior velocidade de emergência ocorrem quando as sementes se encontram entre 1 e $3 \mathrm{~cm}$ de profundidade (Pyon et al, 1977; Martins et al., 2009). Além disso, Pyon et al. (1977) constataram que as sementes de capim-amargoso são mais tolerantes ao estresse hídrico durante a germinação em comparação à Panicum maximum, isto é, as sementes de Digitaria insularis apresentam maior capacidade de germinar e se desenvolver sob condições de 
baixo potencial hídrico do solo em comparação com as plantas de capim-colonião (Panicum maximum).

A emergência de Digitaria insularis e de outras espécies de plantas daninhas foi estudada por Lacerda (2003) por dois anos em sistemas de semeadura direta e convencional. $\mathrm{O}$ autor notou que maiores oscilações na temperatura e na precipitação promoveram maiores fluxos de germinação, fato este que corrobora com as condições ótimas para a germinação descritas por Mondo et al. (2010) e Pyon et al. (1977). No campo, apesar de ter ocorrido emergência de capim-amargoso durante quase o ano todo, o período mais favorável verificado foi o de Fevereiro a Maio (Lacerda, 2003).

\section{Opções de herbicidas para o controle de Digitaria insularis: Pré e Pós- emergência}

Atualmente existem poucos princípios ativos registrados no Brasil para o controle de Digitaria insularis (Tabela 1) e, além disso, excetuando-se o glyphosate, os herbicidas disponíveis são geralmente recomendados para aplicação em pré-emergência ou em estádios precoces de desenvolvimento dessa planta daninha, fato já esperado devido às características de agressividade já discutidas anteriormente.

Quando se trata do controle de Digitaria insularis em pré-emergência, não são relatados problemas na literatura, pois existem vários mecanismos de ação que possuem eficácia sobre capim-amargoso nessa modalidade: inibidores de divisão celular, inibidores do fotossistema II, inibidores da síntese de carotenoides, inibidores da ALS, inibidores da protox. Aliado a isso, ressalta-se que o capim-amargoso possui desenvolvimento inicial lento, sendo facilmente suprimido pela cultura ou mesmo por outras plantas daninhas (Pyon, 1977).
O ponto chave no manejo de Digitaria insularis é que, uma vez estabelecida, a planta se torna muito rústica devido à formação de inúmeros rizomas, e com o conjunto destes, a formação de grandes touceiras. Uma vez ocorrido o processo de perenização, esta planta pode florescer e disseminar sementes com baixos níveis de dormência durante o ano todo. Deste modo, devido à dispersão das sementes pelo vento, os fluxos de emergência desta planta daninha passam a ser dependentes da profundidade em que se encontram as sementes e das condições de umidade do solo.

Para aplicações em pós-emergência, o número de mecanismos de ação se restringe a cinco: temos os inibidores da GS-GOGAT, ACCase, FSI, síntese de carotenoides e EPSPS. No entanto, das cinco opções, apenas três são de herbicidas com caráter sistêmico: os inibidores da síntese de carotenoides, ACCase e EPSPS. Contudo, o glyphosate é o único que não possui restrição de uso em relação ao estádio das plantas de Digitaria insularis, podendo ser utilizado em plantas adultas (florescimento pleno) utilizando-se a dose recomendada.

Os herbicidas inibidores da ACCase atuam em plantas de capim-amargoso em estádios avançados de desenvolvimento, produzindo o sintoma característico de necrose das zonas de crescimento devido ao bloqueio da síntese de lipídeos. Após a aplicação de clethodim sobre uma touceira de capimamargoso em pleno florescimento, é possível remover a haste da panícula facilmente assim como algumas folhas jovens, fato que demonstra a morte do tecido meristemático e a capacidade de translocação desse herbicida.

O problema é que, apesar disso, não ocorre necrose total das folhas da planta, uma vez que apenas as extremidades da folha ficam necrosadas e o restante da folha fica clorótica. Porém, as folhas mantêm-se eretas, ou seja, a aplicação do herbicida não diminui drasticamente a área foliar da planta, a qual 
ainda é capaz de interferir na luminosidade do ambiente onde se encontra.

Os dois mecanismos de ação que apresentam a capacidade de se translocar até os rizomas são os inibidores da EPSPS e os da ACCase, porém, já foram selecionadas populações de capim-amargoso resistentes ao primeiro mecanismo e os herbicidas pertencentes ao segundo não conseguem destruir a parte aérea dessas plantas por completo, apesar de atuarem fortemente nas regiões meristemáticas.

No manejo de Digitaria insularis os herbicidas inibidores do fotossistema I podem suprir parte da lacuna deixada pelos inibidores da ACCase, ou seja, apesar de não serem sistêmicos, estes herbicidas, se empregados corretamente, podem diminuir drasticamente a área foliar dessa planta. Com a perda da área foliar, a planta fica obrigada a utilizar somente suas reservas para emitir novos perfilhos, deste modo, se torna mais sensível aos demais herbicidas e mecanismos de ação empregados em pós-emergência.

No entanto, quando são utilizados somente herbicidas de ação de contato, como [paraquat+diuron] e amônio-glufosinato, em plantas de capim amargoso, é comum que ocorra a diminuição dos níveis de controle com o passar do tempo (Melo et al., 2010). Porque estes herbicidas não são capazes de atuar nos órgãos de reserva e assim, mesmo que as plantas ainda se encontrem com poucos perfilhos, é possível que estas já tenham iniciado a formação de seus rizomas e, portanto, já apresentem a capacidade de emitir novos perfilhos utilizando as reservas armazenadas nos rizomas.

Deste modo, parece claro que as alternativas para o controle de plantas adultas de Digitaria insularis deverão estar baseadas no uso de herbicidas com diferentes mecanismos de ação e estratégias de uso (translocação até os rizomas e destruição da área foliar). Portanto, em muitos casos, o manejo de Digitaria insularis não será realizado com êxito somente com uma única aplicação, já que esta é uma planta de ciclo perene, capaz de se estabelecer durante o ano todo, e as ações de manejo deverão ser realizadas o ano inteiro.

No início do desenvolvimento, as plantas de Digitaria insularis são mais facilmente controladas. Dornelles et al. (2004) verificaram níveis de controle acima de $85 \%$ com a utilização de atrazine, mesotrione e nicosulfuron quando as plantas se encontravam no estádio de 3 a 4 folhas. Já Adegas et al. (2010) demonstraram que empregando clethodim, fluazifop-p-buthyl, fenoxaprop-pethyl, tepraloxydim, [clethodim+fenoxaprop-pethyl], paraquat, haloxyzafop-methyl e imazapyr em plantas de capim-amargoso com até dois perfilhos é possível obter níveis de controle superiores a $90 \%$.

No entanto, quando se trata de plantas em estádio avançado de desenvolvimento (florescidas) observam-se níveis de controle próximos a 50\% com a utilização de [paraquat + diuron], porém com elevada ocorrência de rebrota (Procópio et al., 2006). Situação semelhante foi demonstrada com o uso de mesotrione aplicado em pós-emergência da cultura do milho para o controle da rebrota de plantas de capim-amargoso. Nessa situação este herbicida proporcionou controle próximo a $70 \%$ aos 30 dias após a aplicação, contudo não impediu a formação de grande massa vegetal de capim-amargoso ao final do ciclo da cultura do milho (Timossi et al., 2009).

Ainda são poucos os trabalhos que buscam encontrar definir estratégias de controle para essa planta daninha. Porém, com as informações atualmente disponíveis fica evidente que a utilização pontual de herbicidas inibidores da ACCase em pós-emergência poderá não ser a solução para o problema em todos os estádios de desenvolvimento de Digitaria insularis (Correia \& Durigan, 2009; Parreira et al., 2010).

Além disso, a dependência do uso de um único mecanismo de ação para o controle 
do capim-amargoso, especialmente em áreas de resistência ao glyphosate, pode se constituir numa fonte de pressão de seleção para resistência à ACCase. Com base em trabalhos visando ao controle de plantas já perenizadas, nota-se que o período de utilização de tais misturas poderá apresentar "vida útil" muito pequena devido às altas doses empregadas, e, portanto, alta pressão de seleção (Fornarolli et al., 2011).

Tabela 1. Herbicidas registrados na Secretaria da Agricultura e do Abastecimento do Paraná SEAB - 14/8/2012 para o controle de capim-amargoso (Digitaria insularis).

\begin{tabular}{lcccc}
\hline \multirow{2}{*}{ Herbicidas } & \multicolumn{4}{c}{ Modalidade de Aplicação ${ }^{1 /}$} \\
\cline { 2 - 5 } & PRE & POSi & POSt & POSf \\
\hline Alachlor & $\mathrm{R}^{2 /}$ & $\mathrm{X}$ & $\mathrm{X}$ & $\mathrm{X}$ \\
Amônio-glufosinato & $\mathrm{SI}$ & $\mathrm{R}$ & $\mathrm{R}$ & $\mathrm{SI}$ \\
Clethodim & $\mathrm{X}$ & $\mathrm{R}$ & $\mathrm{R}$ & $\mathrm{SI}$ \\
[Clethodim+Fenoxaprop-p- & $\mathrm{X}$ & $\mathrm{R}$ & $\mathrm{SI}$ & $\mathrm{SI}$ \\
ethyl] & $\mathrm{R}$ & $\mathrm{R}$ & $\mathrm{X}$ & $\mathrm{X}$ \\
Diuron & $\mathrm{X}$ & $\mathrm{R}$ & $\mathrm{R}$ & $\mathrm{R}$ \\
Glyphosate & $\mathrm{R}$ & $\mathrm{X}$ & $\mathrm{X}$ & $\mathrm{X}$ \\
Diuron + Hexazinone & $\mathrm{X}$ & $\mathrm{R}$ & $\mathrm{SI}$ & $\mathrm{SI}$ \\
Mesotrione & $\mathrm{R}$ & $\mathrm{X}$ & $\mathrm{X}$ & $\mathrm{X}$ \\
Pendimenthalin & $\mathrm{X}$ & $\mathrm{R}$ & $\mathrm{SI}$ & $\mathrm{SI}$ \\
Quizalofop-p-ethyl & $\mathrm{X}$ & $\mathrm{R}$ & $\mathrm{SI}$ & SI \\
Quizalofop-p-tefuryl & $\mathrm{X}$ & $\mathrm{R}$ & $\mathrm{SI}$ & SI \\
Sethoxydim & $\mathrm{R}$ & $\mathrm{SI}$ & $\mathrm{SI}$ & SI \\
Trifluralin & $\mathrm{R}$ &
\end{tabular}

II PRE = Herbicida aplicado em pré-emergência da espécie; POSi = Herbicida aplicado em pós-emergência até o segundo perfilho da espécie; POSt $=$ Herbicida aplicado em pós-emergência quando a espécie encontrava-se em perfilhamento pleno; POSf = Herbicida aplicado em pós-emergência quando as plantas apresentam inflorescência; ${ }^{2 /} \mathrm{X}=$ Não recomendado; SI = Sem informação; $\mathrm{R}=$ Recomendado (as informações de época de aplicação são referenciadas nas bulas de cada herbicida disponibilizadas por seus respectivos fabricantes)

\section{Mecanismos de resistência de Digitaria insularis ao glyphosate}

Em virtude de todos os aspectos e condições descritas anteriormente, é compreensível que alguns agricultores possam confundir a maior tolerância a herbicidas em plantas de capim-amargoso após a perenização (formação de rizoma) com a resistência ao herbicida glyphosate, em função da existência de populações com vários níveis de tolerância (Correia et al., 2010) e do aumento crescente na dose de glyphosate requerida para o controle de capim-amargoso com o avanço de seu desenvolvimento (Melo, 2011).
Recentemente foi evidenciada a existência de biótipos de Digitaria insularis realmente resistentes ao glyphosate (Carvalho et al., 2011). Os mecanismos que conferem resistência a essa planta estão relacionados à mais lenta absorção de glyphosate por plantas do biótipo resistente, assim como com a mais rápida metabolização do glyphosate em AMPA, glioxilato e sarcosina. Além disso, a translocação é muito menor em plantas do biótipo resistente em relação ao susceptível, mesmo em plantas novas, com 3 a 4 folhas (Carvalho, 2011). 
A identificação do mecanismo de resistência de Digitaria insularis ao glyphosate (Carvalho, 2011) aliado à comprovação da mudança dos aminoácidos nas posições 182 e 310 da enzima EPSPS de plantas de capimamargoso resistentes (Carvalho et al., 2012) e ao aumento natural da tolerância dessa planta ao glyphosate com o avanço do seu desenvolvimento reforçam a ideia proposta por Neve \& Powles (2005) baseada na resistência de Lolium rigidum a inibidores da ACCase.

Neve \& Powles (2005) concluíram que quando a seleção de um biótipo resistente ocorre pelo uso de doses baixas, os mecanismos de resistência tendem a ser de herança poligênica. Além disso, constataram concomitantemente à seleção de biótipos de Lolium rigidum, o aumento da tolerância desse biótipo a outros herbicidas inibidores da ACCase como também a herbicidas com outros mecanismos de ação. Contudo, ressalta-se que o estudo foi realizado em condições controladas em que os indivíduos selecionados somente cruzavam entre si, sem a entrada de genótipos susceptíveis.

Diferentemente, do que ocorre quando a seleção de biótipos resistentes é realizada pelo uso de doses altas, quando normalmente é verificada uma mutação pontual e muito específica que confere à enzima alvo do herbicidas a insensibilidade ao mesmo, como verificado em alguns biótipos de Lolium multiflorum resistentes a herbicidas inibidores da ACCase (Prado et al., 2000).

Uma vez sabendo-se que os casos de resistência de Digitaria insularis se assemelham aos dos biótipos de Lolium rigidum, deve-se ter em mente que há grande probabilidade de ocorrência de resistência múltipla para essa espécie. Na Tabela 2 há uma compilação dos biótipos de três espécies de gramíneas registradas com histórico de resistência à glyphosate, inibidores da ACCase ou a ambos os mecanismos (Adaptado de Heap, 2011).
Como o glyphosate e os herbicidas inibidores da ACCase são os principais herbicidas usados como graminicidas pósemergentes, é normal que após a ocorrência de um biótipo resistente a um dos mecanismos, o uso do outro aumente intensamente e com isso também a pressão de seleção. Assim, com base nos registros de Heap (2012) foi confeccionada a Tabela 2. Nela foram criados dois parâmetros: o primeiro denominado de T1, que se refere ao período de tempo entre a comprovação de casos de resistência à glyphosate e ACCase isoladamente e o T2, que caracteriza o período de tempo a partir da comprovação da existência de biótipos resistentes aos dois mecanismos isoladamente e a comprovação do surgimento de um biótipo com resistência aos dois mecanismos (resistência múltipla).

A média dos valores de $\mathrm{T} 1$ encontrados é de nove anos, ou seja, estima-se que depois do registro de resistência a um dos mecanismos de ação, a consequente maior pressão de seleção pelo outro mecanismo de ação leve a seleção de biótipos resistentes ao segundo mecanismo em nove anos. Todavia o valor médio para o T2 é de dois anos, o que significa que após a comprovação da existência de biótipos resistentes à glyphosate e a ACCase isoladamente, em média dois anos após, já foi possível encontrar biótipos com resistência múltipla. Esses dados sugerem que basear o manejo de Digitaria insularis resistente ao glyphosate na utilização de herbicidas inibidores da ACCase poder ser uma medida com curto prazo de eficiência.

Ressalta-se que as informações descritas anteriormente se baseiam nos registros de Heap (2012) e são apenas especulações sobre o assunto, visto que não há muito conhecimento com respeito à genética populacional de Digitaria insularis e, portanto faz-se apenas uma análise comparativa com as informações já difundidas sobre as espécies de gramíneas já registradas com resistentes aos mecanismos de ação citados. Contudo, com a 
atual intensificação do uso de herbicidas é de maneira mais frequente no futuro próximo. possível que os eventos de resistência ocorram

Tabela 2. Compilação dos casos de comprovação de resistência de gramíneas à glyphosate e herbicidas inibidores da ACCase registrados no mundo (Adaptado a partir de Heap, 2012).

\begin{tabular}{|c|c|c|c|c|c|c|}
\hline \multirow{2}{*}{ Nome científico } & \multicolumn{4}{|c|}{ Registro de casos de resistência } & T1 & $\overline{\mathrm{T} 2}$ \\
\hline & Local & ACCase & Glyphosate & Múltipla & \multicolumn{2}{|c|}{$\operatorname{Ano}(\mathbf{s})$} \\
\hline Eleusine indica & Malásia & 1990 & - & 1997 & 7 & 0 \\
\hline Eleusine indica & Bolívia & 2005 & - & - & - & - \\
\hline Eleusine indica & Brasil & 2003 & - & - & - & - \\
\hline Eleusine indica & Colômbia & - & 2006 & - & - & - \\
\hline Eleusine indica & Estados Unidos & - & 2010 & - & - & - \\
\hline L. multiflorum & Chile & 1998 & 2001 & 2006 & 3 & 5 \\
\hline L. multiflorum & Argentina & 2009 & 2007 & 2010 & 2 & 1 \\
\hline L. multiflorum & Brasil & - & 2003 & 2010 & 7 & 0 \\
\hline L. multiflorum & França & 1993 & - & - & - & - \\
\hline L. multiflorum & Itália & 1995 & - & - & - & - \\
\hline L. multiflorum & Espanha & - & 2006 & - & - & - \\
\hline L. multiflorum & Reino Unido & 1990 & - & - & - & - \\
\hline L. multiflorum & Estados Unidos & 1987 & 2004 & - & 17 & - \\
\hline L. rigidum & Austrália & 1984 & 1996 & 1999 & 8 & 3 \\
\hline L. rigidum & Israel & 1998 & - & 2007 & 9 & 0 \\
\hline L. rigidum & África do Sul & 1993 & 2001 & 2003 & 8 & 2 \\
\hline L. rigidum & Chile & 1997 & - & - & - & - \\
\hline L. rigidum & França & 1993 & 2005 & - & 12 & - \\
\hline L. rigidum & Iran & 2007 & - & - & - & - \\
\hline L. rigidum & Itália & - & 2007 & - & - & - \\
\hline L. rigidum & Arábia Saudita & 1992 & - & - & - & - \\
\hline L. rigidum & Espanha & 1992 & 2006 & - & 14 & - \\
\hline L. rigidum & Tunísia & 1996 & - & - & - & - \\
\hline L. rigidum & Estados Unidos & - & 1998 & - & - & - \\
\hline Média aproximada & & & & & 9 & 2 \\
\hline
\end{tabular}

T1: Período de tempo entre a comprovação de casos de resistência para ambos os mecanismos isoladamente (Glyphosate e ACCase); T2 Período de tempo a partir da comprovação da existência de biótipos resistentes aos dois mecanismos isoladamente e a comprovação do surgimento de um biótipo com resistência aos dois mecanismos (resistência múltipla).

\section{Perspectivas para o manejo de capim- amargoso}

Diante dos fatos, ideias e conceitos apresentados nesta revisão, é possível prever que a recomendação para o manejo de capim- amargoso (Digitaria insularis) não será única e pontual, pois a determinação das melhores práticas envolverá o conhecimento sobre o comportamento dessa planta daninha o ano inteiro, assim como, os resultados de eficácia 
dos herbicidas sobre a mesma em todos os seus estádios de desenvolvimento e épocas do ano.

Novamente, o capim-amargoso é uma planta perene que tem a capacidade de germinar, crescer e se desenvolver praticamente $\mathrm{o}$ ano inteiro. Uma vez estabelecida, com a formação de rizomas, a dificuldade de controle dessa espécie aumenta muito. Assim, os agricultores e técnicos deverão estar atentos a presença dessa espécie durante a safra, safrinha e entressafra, pois o descaso com a ocorrência dessa planta na lavoura pode levar a um forte aumento na população de Digitaria insularis e consequentemente a sérios prejuízos na produtividade e aumento nos custos de produção.

\section{Referências}

ADEGAS, F.S. et al. Alternativas de controle químico de Digitaria insularis resistente ao herbicida glyphosate. In: CONGRESSO BRASILEIRO DA CIÊNCIA DAS PLANTAS DANINHAS, 27., 2010, Ribeirão Preto. Resumos... Ribeirão Preto: Sociedade Brasileira da Ciência das Plantas Daninhas, 2010.

BRACCINI, A.L. Bancos de semente e mecanismos de dormência em sementes de plantas daninhas. In: OLIVEIRA JR., R.S.; CONSTANTIN, J.; INOUE, M.H. (Eds.). Biologia e manejo de plantas daninhas. Curitiba: Omnipax Editora, 2011. p.37-66.

CANTO-DOROW, T.S. Digitaria Heister ex Haller. In: WANDERLEY, M.G.L.; SHEPHERD, G.J.; GIULIETTI, A.M. (Ed.) Flora fanerogâmica do Estado de São Paulo. São Paulo: HUCITEC, 2001. p.143-150.

CARVALHO L.B. et al. Pool of resistance mechanisms to glyphosate Digitaria insularis. Journal of Agricultural and Food Chemistry, v.60, n.2, p.615-622, 2012.

CARVALHO, L.B. et al. Detection of sourgrass (Digitaria insularis) biotypes resistant to glyphosate in Brazil. Weed Science, v.59, n.2, p.171-176, 2011.

CARVALHO, L.B. et al. Estudo comparativo do acúmulo de massa seca e macronutrientes por plantas de milho var. BR-106 e Brachiaria plantaginea. Planta Daninha, v.25, n.2, p.293301, 2007.

CARVALHO, L.B. Interferência de Digitaria insularis em Coffea arabica e respostas destas espécies ao glyphosate. 2011. 119f. Tese (Doutorado em Agronomia) Universidade Estadual Paulista "Júlio de Mesquita Filho" Faculdade de Ciências Agrárias e Veterinárias, Jaboticabal.

CASTRO, P.R.C.; FERREIRA, S.O.; YAMADA, T. Ecofisiologia da produção agrícola. Piracicaba: POTAFOS, 1987. 249p.

CLAYTON, W.D. et al. (2006). Grass Base The Online World Grass Flora. http://www.kew.org/data/grasses-db.html. \{acessado 16 de Maio 2011\}.

CORREIA, N.M.; DURIGAN, J.C. Manejo químico de plantas adultas de Digitaria insularis (L.) Fedde com glyphosate isolado e em mistura com chlorimuronethyl ou quizalofop-p-tefuril em área de plantio direto. Bragantia, v.68, n.3, p.689-697, 2009.

CORREIA, N.M.; LEITE, G.J.; GARCIA, L.D. Resposta de diferentes populações de Digitaria insularis(L.) Fedde ao herbicida glyphosate. Planta Daninha, v.28, n.4, p.769776, 2010.

DORNELLES, S.H.B. et al. Controle de plantas daninhas do gênero Digitaria sp. com o herbicida mesotrione na cultura do milho (Zea mays). In: CONGRESSO BRASILEIRO DA CIÊNCIA DAS PLANTAS DANINHAS, 24., 2004, São Pedro. Resumos... São Pedro: Sociedade Brasileira da Ciência das Plantas Daninhas, 2004. p.107.

FORNAROLLI, D.A. et al. Manejo de biotípos de Digitaria insularis resistente ao herbicida glifosato. In: III SIMPÓSIO 
INTERNACIONAL SOBRE GLYPHOSATE, Botucatu, SP, 2011. Resumos... Botucatu:UNESP, 2011.

HEAP, I.M. International Survey of Herbicide Resistant Weeds. Online http://www.weedscience.com. Acesso em 14 de Junho de 2012.

KISSMANN, K.G.; GROTH, D. Plantas infestantes e nocivas. 2.ed. São Paulo: BASF, 1997. Tomo I. 825 p.

LACERDA, A.L.S. Fluxos de emergência e banco de sementes de plantas daninhas em sistema de semeadura direta e convencional e curvas dose-resposta ao glyphosate. 2003. $153 \mathrm{f}$. Tese (Doutorado em Fitotecnia) - Escola Superior de Agricultura Luiz Queiroz, Universidade de São Paulo, Piracicaba.

LORENZI, H. Plantas daninhas do Brasil: terrestres, aquáticas, parasitas e tóxicas. 3.ed. Nova Odessa: Plantarum, p. 608, 2000.

MACHADO, A.F.L. et al. Caracterização anatômica de folha, colmo e rizoma de Digitaria insularis (L.) Fedde. Planta Daninha, v.26, n.1, p.1-8, 2008.

MACHADO, A.F.L. et al. Análise de crescimento de Digitaria insularis (L.) Fedde. Planta Daninha, v.24, n.4, p.641-647, 2006

MARTINS J.F. et al. Efeito da profundidade de semeadura na emergência do capim-amargoso (Digitaria insularis (L.) Fedde). In: CONGRESSO DE INICIAÇÃO CIENTÍFICA DA UNESP (CIC), 21., São José do Rio Preto, SP, 2009. Resumos... São José do Rio Preto: UNESP, 2009.

MELO, M.S.C. Alternativas de controle, acúmulo de chiquimato e curva de crescimento de capim-amargoso (Digitaria insularis) suscetível e resistente ao glyphosate. 2011. 74f. Dissertação (Mestrado em Fitotecnia) - Escola Superior de Agricultura Luiz de Queiroz, Universidade de São Paulo, Piracicaba, 2011.
MELO, M.S.C. et al. Alternativas de controle para capim-amargoso (Digitaria insularis) resistente ao glifosato. In: CONGRESSO BRASILEIRO DA CIÊNCIA DAS PLANTAS DANINHAS, 27.,2010, Ribeirão Preto. Resumos... Ribeirão Preto: Sociedade Brasileira da Ciência das Plantas Daninhas, 2010.

MONDO, V.H.V. et al. Efeitos da luz e temperatura na germinação de sementes de quatro espécies de plantas daninhas do gênero Digitaria. Revista Brasileira Sementes, v. 32, n.1, p.131-137, 2010.

NEVE, P. \& POWLES, S. Recurrent selection with reduced herbicide rates results in the rapid evolution of herbicide resistance in Lolium rigidum. Theoretical and Applied Genetics, v.110, n.6, p.1154-1166, 2005.

PARREIRA, M.C. et al. Manejo químico de Digitaria insularis em área de plantio direto. Revista Brasileira Ciências Agrárias, v.5, n.1, p.13-17, 2010.

PRADO, R. et al. Resistance to acetylCoAcarboxylase-inhibiting herbicides in Lolium multiflorum. Weed Science,v.48, n.3, p.311-318, 2000.

PROCÓPIO, S.O. et al. Efeitos de dessecantes no controle de plantas daninhas na cultura da soja. Planta Daninha, v.24, n.1, p.193-197, 2006.

PYON, J.Y. Studies on the biology of sourgrass (Trichachne insularis (L.) Nees and of its competition with buffelgrass (Cenchrus ciliaris L.) and guineagrass (Panicum maximum Jacq.). 1975. 133p. Thesis (Doctor of Philosophy in agronomy and Soil Science) - University of Hawaii.

PYON, J.Y., WHITNEY, A.S., NISHIMOTO, R.K. Biology of sourgrass and its competition with buffelgrass and guineagrass. Weed Science, v.25, n.2, p.171-174, 1977.

SEAB. Secretaria da Agricultura e do Abastecimento do Paraná - SEAB. Disponível 
em: http://celepar07web.pr.gov.br/agrotoxicos/. Acesso em: 14 de junho de 2012.

SILVA, A.C. et al. Análise de crescimento de Brachiaria brizantha submetida à doses reduzidas de fluazifop-p-butil. Planta Daninha, v.3, n.1, p.85-91, 2005.

TAIZ, L.; ZEIGER, E. Fisiologia vegetal. 3ed. Porto Alegre: Artmed, 2004.

TIMOSSI, P.C. et al. Manejo de rebrotes de Digitaria insularis (L.) Fedde no plantio direto de milho. Planta Daninha, v.27, n.1, p.175179, 2009. 